\title{
Temperature Dependence of the Zero-Bias Conductance in the Graphene NIS Junction
}

\author{
M.M. WYSOKIŃSKI*
}

Marian Smoluchowski Institute of Physics, Jagiellonian University, W.S. Reymonta 4, 30-059 Kraków, Poland

\begin{abstract}
The temperature dependence of the zero-bias conductance of the graphene-based, ballistic junction composed of the three consecutive regions: normal, with potential barrier ("insulating") and superconducting (NIS), is analyzed within the extended Blonder-Tinkham-Klapwijk approach. Within this approach we have found that oscillatory behavior of the conductance as a function of barrier strength is suppressed by the temperature - the amplitude diminishes with heating up the junction. Moreover, the subtle, although nontrivial feature of the system is reported: the average over the period of the oscillations of the zero-bias conductance for relatively small Fermi level mismatch behaves non-monotonically with the increase of the temperature with the maximum roughly at $T / T_{\mathrm{c}} \approx 0.5$.
\end{abstract}

DOI: 10.12693/APhysPolA.126.A-36

PACS: $74.45 .+\mathrm{c}, 73.23$.Ad

\section{Introduction}

The discovery of the graphene [1] opened up new opportunities in the material science for studying and designing electronic systems governed by relativistic quantum electrodynamics in the desktop laboratories. Shortly after, the field of quasi-relativistic physics has been enriched by the range of new materials where similar electronic structure to the graphene was found, such as silicon and germanium atoms arranged in a hexagonal lattice [2-4], and topological insulators [5] (surface states).

Despite similar energy dispersion, most of those new systems turned out to be significantly different from graphene. Especially, silicene was found to be intrinsically superconducting [6], contrary to the graphene, which has widened the freedom of experimental and theoretical studies on the superconducting, graphene-like systems.

One of the first concepts in this subfield was the junction based on the graphene, composed from normal and superconducting (induced by means of proximity effect) regions, proposed by Beenakker in his seminal work [7]. He found that due to the quasi-relativistic nature of the charge carriers, the conductance spectra differ significantly from the standard case [8]. Later, also other, more sophisticated ideas of electronic devices based on the interplay of the Dirac physics with the superconductivity were proposed [9-11].

In majority of articles concerning transport properties through the graphene-based superconducting hybrid structures the well-established extended BlonderTinkham-Klapwijk (BTK) formalism [8] has been used. Particularly this approach was proven to be successful for this class of systems in calculating e.g. conductance spectra $[7,12-14]$, heat conductance $[9,15-17]$ as well as thermopower [18] with the density of states explicitly

\footnotetext{
*e-mail: marcin.wysokinski@uj.edu.pl
}

taken into account [19].

Within this approach, the oscillatory behavior of the electric, as well as the heat conductance, as a function of the barrier strength was found in the graphene-based junction composed of normal and superconducting regions with the high external potential at the interface (NIS junction) [12, 13, 15]. This peculiar property of this system is a direct manifestation of the quasi-relativistic nature of charge carriers in the graphene.

The natural question that arises at this stage, that in particular we address in this work, is how the oscillatory behavior of the zero-bias conductance (ZBC) as a function of the barrier strength is affected by the increasing temperature. Particularly we have investigated temperature dependence of the amplitude, and the average over one period of the oscillations for different Fermi level mismatch (FLM) between normal and superconducting part of the junction.

The paper is organized as follows. In Sect. 2 we present briefly generalized BTK formalism for the calculating current through the NIS junction in the non-zero temperature. Then in Sect. 3 we discuss our results, mainly focused on the behavior of the conductance oscillations as a function of barrier strength with the raising temperature. Finally, we conclude in Sect. 4.

\section{Model}

We consider graphene-based junction composed of normal and superconducting regions with the energy potential of the width $d$ and the height $V_{0}$ at the interface playing the role of the "insulating" barrier. Our starting point in the description of our system are Dirac-Bogoliubovde Gennes equations for the two-dimensional (2D) sheet of graphene in the form [7, 20]:

$$
\left(\begin{array}{cc}
\mathcal{H}_{j}-E_{\mathrm{F}} \mathbb{1}+U(\boldsymbol{r}) \mathbb{1} & \Delta \\
\Delta^{\dagger} & E_{\mathrm{F}} \mathbb{1}-U(\boldsymbol{r}) \mathbb{1}-\mathcal{H}_{j}
\end{array}\right) \psi=\epsilon \psi,
$$

where the index $j$ can be either + or - which refers to the two inequivalent valleys $K$ and $K^{\prime}$ in the Brillouin zone, and the single particle Hamiltonian reads 


$$
\mathcal{H}_{j}=-\mathrm{i} \hbar v_{\mathrm{F}}\left(\sigma_{x} \partial_{x}+\operatorname{sgn}(j) \sigma_{y} \partial_{y}\right),
$$

with $v_{\mathrm{F}}$ standing for momentum independent velocity of charge carriers in graphene, and $\sigma_{i}$ \} denoting respective Pauli matrices. The potential $U(\boldsymbol{r})$ in Eq. (1) shifts Fermi energies in the normal, "insulating" and superconducting region and can be modeled as

$$
U(\boldsymbol{r})=-U_{0} \theta(x)+V_{0} \theta(-x) \theta(x+d) .
$$

We would treat the potential barrier at the interface of the junction in the limit of thin barrier $(d \rightarrow 0)$ and high potential $\left(V_{0} \rightarrow \infty\right)$, at the same time keeping effective dimensionless barrier strength $\chi$ constant, defined similarly as in Refs. $[12,13,15]$ as

$$
\chi=\frac{V_{0} d}{\hbar v_{\mathrm{F}}} .
$$

The potential $U_{0}$ is used to tune effective Fermi level mismatch between normal and superconducting regions of the graphene sheet. In order to satisfy the meanfield condition for the superconductivity we set $\Delta_{0} \ll$ $\left(U_{0}+E_{\mathrm{F}}\right)$. Note that FLM can be also a source of the normal reflection. We have assumed the pairing potential with the $s$-wave symmetry changing step-like at the interface and having temperature dependence deduced from the usual BCS theory [21],

$$
\Delta(\boldsymbol{r}, T)=\Delta_{0} \theta(x) \tanh \left(1.76 \sqrt{\frac{T_{\mathrm{c}}}{T}-1}\right) .
$$

Following the BTK formalism one should identify the relevant scattering processes and construct full wave functions for the normal $\left(\Psi_{\mathrm{N}}\right)$, "insulating" $\left(\Psi_{\mathrm{I}}\right)$ and superconducting $\left(\Psi_{\mathrm{S}}\right)$ regions, and force their continuity at the interfaces $[12,13]$. Incoming electron $\left(\psi_{+}^{\mathrm{e}}\right)$ from the normal side of the graphene sheet can be backscattered either in the normal reflection process $\left(\psi_{-}^{e}\right)$ or in so-called Andreev reflection process as a converted by the pair potential hole $\left(\psi_{-}^{\mathrm{h}}\right)$. In turn, in terms of the transmission it can be injected into the superconducting region as a Bogoliubov quasiparticle $\left(\psi_{+}^{\mathrm{Se}}, \psi_{-}^{\mathrm{Sh}}\right)$. There are also bound electron and hole states in the "insulating" region $\left(\psi_{-}^{\text {Ie }}\right.$, $\left.\psi_{+}^{\mathrm{Ie}}, \psi_{-}^{\mathrm{Ih}}, \psi_{+}^{\mathrm{Ih}}\right)$. The respective full wave functions can be written in the following form:

$$
\begin{aligned}
& \Psi_{\mathrm{N}}=\psi_{+}^{\mathrm{e}}+r \psi_{-}^{\mathrm{e}}+r_{a} \psi_{-}^{\mathrm{h}}, \\
& \Psi_{\mathrm{I}}=a_{\mathrm{e}} \psi_{+}^{\mathrm{Ie}}+a_{\mathrm{h}} \psi_{+}^{\mathrm{Ih}}+b_{\mathrm{e}} \psi_{-}^{\mathrm{Ie}}+b_{\mathrm{h}} \psi_{-}^{\mathrm{Ih}}, \\
& \Psi_{\mathrm{S}}=t_{\mathrm{e}} \psi_{+}^{\mathrm{Se}}+t_{\mathrm{h}} \psi_{-}^{\mathrm{Se}} .
\end{aligned}
$$

Under the condition of the continuity at the boundaries of the global wave function,

$$
\left.\Psi_{\mathrm{N}}\right|_{x=-d}=\left.\Psi_{\mathrm{I}}\right|_{x=-d},\left.\quad \Psi_{\mathrm{I}}\right|_{x=0}=\left.\Psi_{\mathrm{S}}\right|_{x=0},
$$

one can derive respective wave functions amplitudes. Note that contrary to the standard case [8], due to the linearity of the Dirac-Weyl equation there is no need to match also derivatives of wave functions. The direct energy and angle of incidence dependence of the needed amplitudes $r$ and $r_{a}$ can be obtained after some straightforward algebra and are not shown here explicitly. For full formula and also explicit particles and quasi-particles wave function forms see e.g. Refs. [12, 13, 15].
The transmission probability through the NIS junction now can be expressed as [8]:

$$
\begin{aligned}
& \mathcal{T}(\epsilon)=\int_{-\pi / 2}^{\pi / 2} \mathrm{~d} \theta \frac{\cos \theta}{2} \\
& \times\left(1-|r(\epsilon, \theta)|^{2}+\frac{\operatorname{Re}\left[\mathrm{e}^{\mathrm{i} \theta_{A}}\right]}{\cos \theta}\left|r_{a}(\epsilon, \theta)\right|^{2}\right) .
\end{aligned}
$$

With the knowledge of the transmission probability in the spirit of the Landauer formalism one can write a full formula for the ballistic charge transport through the junction,

$$
\begin{gathered}
I_{\mathrm{e}}(V)=\frac{4 e}{h} \int_{-\infty}^{\infty} \mathrm{d} \epsilon N(\epsilon) \mathcal{T}(\epsilon) \\
\times\left(f^{\mathrm{N}}(\epsilon-e V)-f^{\mathrm{S}}(\epsilon)\right),
\end{gathered}
$$

where functions $f^{(i)}$ denote Fermi-Dirac distributions with the superscript $\{i=\mathrm{N}, \mathrm{S}\}$ standing for the normal and superconducting region, respectively. The formula (9) holds for the biased junction with the voltage, $V$ and accounts for the density of states in the graphene sheet of the width $W$,

$$
N(\epsilon)=\frac{\left|E_{\mathrm{F}}+\epsilon\right| W}{\pi \hbar v_{\mathrm{F}}} .
$$

The ZBC now can be obtained in a straightforward manner by linearizing Fermi functions with respect to the small voltage $(V \rightarrow 0)$,

$$
G=\frac{\partial I}{\partial V}=-\frac{4 e^{2}}{h} \int_{-\infty}^{\infty} \mathrm{d} \epsilon \frac{\partial f}{\partial \epsilon} N(\epsilon) \mathcal{T}(\epsilon) .
$$

It is convenient to normalize above formula by the ballistic conductance of the same sheet of graphene without superconducting or external potential sources, kept in the same temperature,

$$
g_{0}=-\frac{4 e^{2}}{h} \int_{-\infty}^{\infty} \mathrm{d} \epsilon \frac{\partial f}{\partial \epsilon} N(\epsilon) .
$$

\section{Results}

In all numerical calculations we have set shifted Fermi level in the superconducting region as $E_{\mathrm{F}}^{\prime}=E_{\mathrm{F}}+U_{0}=$ $100 \Delta_{0}$.

The theoretical prediction of the oscillatory behavior of ZBC spectra (cf. Fig. 1) as a function of the effective barrier strength has been reported in the graphene NIS junction some time ago [12]. The issue mainly investigated here is how this quasi-relativistic behavior changes with increasing temperature. Therefore, we have analyzed the effect of the temperature, the effective barrier strength and the FLM on the normalized zero-bias conductance through the graphene NIS junction (calculated from Eq. (11)).

For $E_{\mathrm{F}}^{\prime} / E_{\mathrm{F}} \geq 10$ the oscillations almost completely disappear (cf. Fig. 1), and in fact the effect of the barrier strength is negligible in this regime. Therefore in this limit NIS junction properties are similar to that of the NS junction in which case ZBC behaves non-trivially with the raising temperature (see Ref. [18]).

In the opposite regime, for $E_{\mathrm{F}}^{\prime} / E_{\mathrm{F}} \leq 10$, by changing the barrier strength parameter, $\chi$ we can control to some 


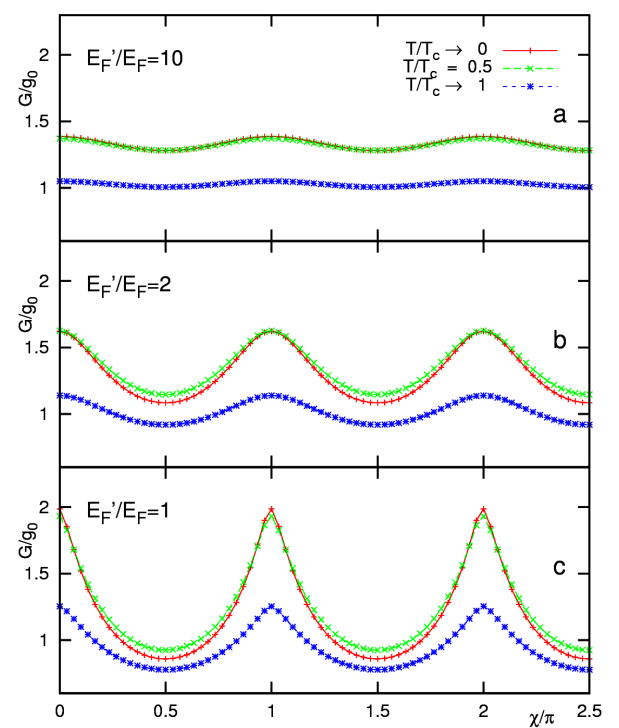

Fig. 1. Normalized ZBC as a function of the effective barrier strength, $\chi$ for selected temperatures. The plots in parts (a), (b), (c) differ with the FLM. The amplitude of the oscillations diminishes with the increase of the temperature (cf. inset in Fig. 3). Note that the average value of ZBC can slightly increase (cf. Fig. 3). At part (a) plots for $T / T_{c} \rightarrow 0$ and $T / T_{c}=0.5$ are mostly overlapping.

larger extent the mutual relation between the probability of the Andreev and the normal reflection. However, with the increasing temperature the range of the manipulation diminishes which has a direct manifestation in the monotonic decrease of the ZBC oscillations amplitude (cf. inset of Fig. 3).

What is more, the upper limit of the oscillations (where the Andreev reflection dominates) is relatively less affected by the increase of the temperature than bottom limit approximately up to $T \approx 0.5 T_{\mathrm{c}}$ which is smooth boundary designated by the regime where thermal excitation are within the range of the superconducting gap. The situation reverses when the superconducting gap starts closing. This in turn gives rise to a non-monotonic change of the average $\mathrm{ZBC}$, defined as:

$$
G_{\mathrm{av}}=\frac{1}{\pi} \int_{0}^{\pi} G(\chi) \mathrm{d} \chi
$$

which in the low FLM regime $\left(E_{\mathrm{F}}^{\prime} / E_{\mathrm{F}} \leq 10\right)$ acquires maximum around $T \approx 0.5 T_{c}$ (cf. Fig. 3 ). In comparison, ZBC of the graphene-based NS junction also has a nonmonotonic behavior with the raising temperature but in the different regimes (i.e. for small position of the Fermi level in normal region of the junction) and of the different kind (cf. Ref. [18]). Also the origin of this behavior can be seen in the evolution of the ZBC with the increase of the temperature (cf. Fig. 2). For selected values of the barrier strength from the first period $\left(\chi=\frac{\pi}{2}, \frac{\pi}{4}\right) \mathrm{ZBC}$ is increasing up to a flat maximum approximately at the $T / T_{\mathrm{c}} \approx 0.5$ (cf. Fig. 2b, c). In the same regime for $\chi=0$

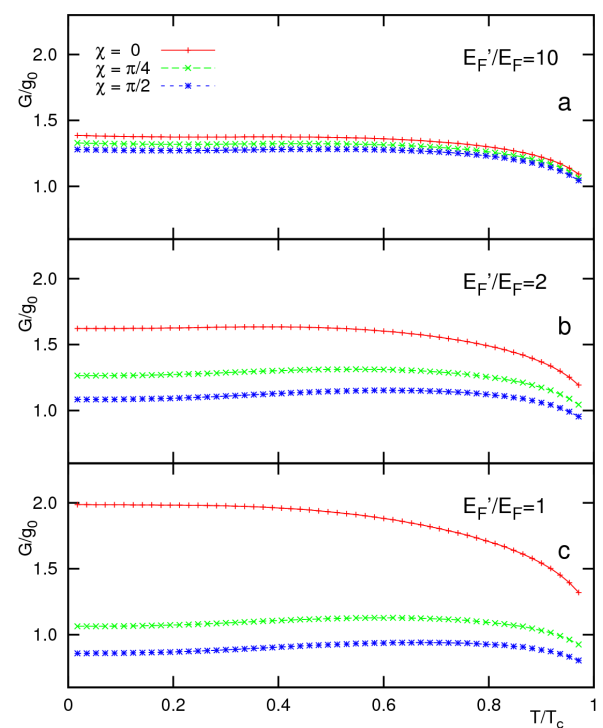

Fig. 2. Normalized ZBC as a function of the temperature for selected barrier strengths, $\chi$. The plots in parts (a), (b), (c) differ with the FLM. For specific values of barrier strength, we obtain flat maximum (parts (b), (c)).

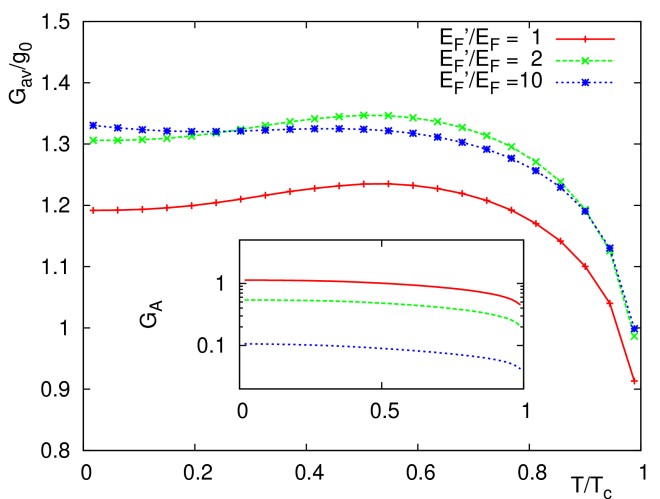

Fig. 3. The main plot presents averaged $\mathrm{ZBC}$ over the period of oscillations with respect to the change of the barrier strength as a function of the temperature for selected values of the FLM. In the inset we plot with the logarithmic scale the change of the oscillations amplitude $\left(G_{A}\right)$ also as a function of the temperature. For relatively small FLM, the average $\mathrm{ZBC}$ changes nonmonotonically with the temperature and has a maximum around $T / T_{c} \approx 0.5$, contrary to the monotonic decrease of the amplitude of ZBC oscillations.

it drops slowly and remains almost unchanged.

\section{Conclusions}

In this work we have analyzed the temperature dependence of the zero-bias conductance of the graphene-based NIS ballistic junction. We have found that with the increasing temperature oscillations of the zero-bias conductance as a function of the barrier strength persist with 
the monotonically decreasing amplitude. The interesting feature of the system is a non-monotonic change, of the average over the period of the oscillations of the zero-bias conductance (as a function of a barrier strength) with the increase of the temperature, with the maximum around $T / T_{\mathrm{c}} \approx 0.5$ in the limit of a relatively small Fermi level mismatch $\left(E_{\mathrm{F}}^{\prime} / E_{\mathrm{F}} \lesssim 10\right)$.

\section{Acknowledgments}

The work has been supported by the Foundation for Polish Science (FNP) under the TEAM program. The author also greatly acknowledge financial support from the project DOCTUS.

\section{References}

[1] K.S. Novoselov, A.K. Geim, S.V. Morozov, D. Jiang, M.I. Katsnelson, I.V. Grigorieva, S.V. Dubonos, A.A. Firosov, Nature 438, 197 (2005).

[2] G.G. Guzmán-Verri, L.C. Lew Yan Voon, Phys. Rev. $B$ 76, 075131 (2007).

[3] S. Lebègue, O. Eriksson, Phys. Rev. B 79, 115409 (2009).

[4] S. Cahangirov, M. Topsakal, E. Aktürk, H. Sahin, S. Ciraci, Phys. Rev. Lett. 102, 236804 (2009).

[5] X.-L. Qi, S.-C. Zhang, Rev. Mod. Phys. 83, 1057 (2011).

[6] L. Chen, B. Feng, K. Wu, Appl. Phys. Lett. 102, 081602 (2013).
[7] C.W.J. Beenakker, Phys. Rev. Lett. 97, 067007 (2006).

[8] G.E. Blonder, M. Tinkham, T.M. Klapwijk, Phys. Rev. B 25, 4515 (1982).

[9] M. Titov, C.W.J. Beenakker, Phys. Rev. B 74, 041401 (2006).

[10] M. Titov, A. Ossipov, C.W.J. Beenakker, Phys. Rev. $B$ 75, 045417 (2007).

[11] S.-G. Cheng, Y. Xing, J. Wang, Q.-F. Sun, Phys. Rev. Lett. 103, 167003 (2009).

[12] S. Bhattacharjee, K. Sengupta, Phys. Rev. Lett. 97, 217001 (2006).

[13] J. Linder A. Sudbø, Phys. Rev. B 77, 064507 (2008).

[14] Q. Zhang, D. Fu, B. Wang, R. Zhang, D.Y. Xing, Phys. Rev. Lett. 101, 047005 (2008).

[15] T. Yokoyama, J. Linder, A. Sudbø, Phys. Rev. B 77, 132503 (2008).

[16] M. Salehi, M. Alidoust, G. Rashedi, J. Appl. Phys. 108, 083917 (2010).

[17] M. Salehi, M. Alidoust, Y. Rahnavard, G. Rashedi, J. Appl. Phys. 107, 123916 (2010).

[18] M. Wysokiński, J. Spałek, J. Appl. Phys. 113, 163905 (2013).

[19] M.M. Wysokiński, Acta Phys. Pol. A 122, 758 (2012).

[20] P.G. de Gennes, Superconductivity in Metals and Alloys, W. A. Benjamin, New York 1966, Ch. 5.

[21] J.B. Ketterson, S.N. Song, Superconductivity, Cambridge University Press, Cambridge 1999. 